\title{
Global Contrast in Nuclear Medicine
}

A

"it's like comparing apples and oranges" was widely used when arguing the relative merits of ${ }^{99 \mathrm{~m}} \mathrm{Tc}$-based radiopharmaceuticals with the more traditional ${ }^{201}$ Tl-chloride in myocardial perfusion imaging. Recent articles in the Journal of Nuclear Medicine Technology (JNMT) highlighted that this same expression might be equally relevant when discussing the provision of nuclear medicine services across the developed world.

For many years, developed countries in the northern hemisphere have enjoyed a reliable and cost-effective supply of cyclotron-produced radionuclides, whereas those in the southern hemisphere, particularly Australia, relied on importing cyclotron-produced

See J Nucl Med Technol 2010; $38: 1-3,6-17$

nuclides from the northern hemisphere. In the early 1990s, the Australian National Medical Cyclotron was constructed to meet the local needs for cyclotron-produced PET and general nuclear medicine radionuclides. Unfortunately, the investment failed on both fronts, and the system was commercially decommissioned this year, leaving Australia with only a handful of compact cyclotrons dedicated to PET tracer production. Although cyclotronproduced radionuclides are readily and cost-effectively available in the northern

Received Apr. 27, 2010; revision accepted Jul. 8, 2010.

For correspondence or reprints contact: Geoff Currie, Australian School of Advanced Medicine, Macquarie University, P.O. Box U102, Sydney, Australia.

E-mail: gcurrie@csu.edu.au

COPYRIGHT (C) 2010 by the Society of Nuclear Medicine, Inc.

DOI: 10.2967/jnmt.110.078535 hemisphere (e.g., ${ }^{123}$ I-metaiodobenzylguanidine), the southern hemisphere is confronted with a relative cost-prohibition. Indeed, although most nuclear medicine texts cite the importance of ${ }^{123}$ I in thyroid imaging, it is not generally used in Australia.

The global ${ }^{99 \mathrm{~m}} \mathrm{Tc}$ crisis, however, should be more accurately described as a compartmented global crisis because the impact in the southern hemisphere has been minimal. Dated reactors and lack of new reactor installations in the northern hemisphere will produce recurring ${ }^{99 \mathrm{~m}} \mathrm{Tc}$ supply stress. Indeed, the Society of Nuclear Medicine Technologist Section (SNMTS) recently launched an awareness campaign in an attempt to protect the ${ }^{99 m}$ Tc-based imaging industry. The "Got ${ }^{99 m}$ Tc?" T-shirt is part of that initiative. Furthermore, cyclotrons are being looked at to produce ${ }^{99 \mathrm{~m}} \mathrm{Tc}$ more reliably (1), which casts the industry back to the pre-- ${ }^{99} \mathrm{Mo} /{ }^{99 \mathrm{~m}} \mathrm{Tc}$ generator era of daily deliveries, unreliable service (particularly after hours), and carrier-related issues with image quality. Changes to clinical practice have been proposed to accommodate the lack of ${ }^{99 \mathrm{~m} T c}$. Because of this lack of ${ }^{99 \mathrm{~m}} \mathrm{Tc}$, Pagnanelli and Basso reported in a recent JNMT article (2) on the clinical utility and protocols for ${ }^{201} \mathrm{Tl}$ myocardial perfusion studies, a regression to days gone by. The use of ${ }^{67} \mathrm{Ga}$-citrateand ${ }^{111} \mathrm{In}$-labeled white blood cells in preference to ${ }^{99 \mathrm{~m}} \mathrm{Tc}$-labeled white blood cells in infection imaging provides another example. In the same journal, Buck et al. (3) reported that the proliferation of PET/CT in the United States was well in excess of global trends, perhaps a measure of funding policy and an adoption of technology not reliant on ${ }^{99 \mathrm{~m}} \mathrm{Tc}$ supply. With reference to the former, because of the much more prescriptive and restrictive policy in Australia, the
United States enjoys a much greater clinical diversity of rebates than does Australia. It would be an interesting exercise to gain a snapshot of whether the global ${ }^{99 \mathrm{~m}} \mathrm{Tc}$ shortage has seen an associated increase in the number of ${ }^{18} \mathrm{~F}$ ion bone scans or, in fact, a tendency to favor a move toward ${ }^{13} \mathrm{~N}$ ammonia myocardial perfusion more than a regression toward ${ }^{201} \mathrm{Tl}$. One consolation in this scenario is that patients are retained in nuclear medicine. Anecdotal evidence from the United Kingdom suggests that, for example, bone scanning may be performed only on a single day per week, which has encouraged a more widespread reliance on anatomic imaging. At a time when anatomic imaging is challenging traditional strongholds of nuclear medicine (e.g., cardiac CT or MRI and CT angiography for pulmonary embolism), the lack of ${ }^{99 \mathrm{~m}} \mathrm{Tc}$ provides little resistance.

In the southern hemisphere, there could not be a greater contrast. There are numerous new reactors, including in Australia and South America, and high-production capabilities in South Africa. There is no shortage of ${ }^{99 \mathrm{~m}} \mathrm{Tc}$, and indeed, it is used in preference to cyclotron-produced radionuclides such as ${ }^{67} \mathrm{Ga}$-citrate and ${ }^{201} \mathrm{Tl}$. The hemispheric gap is perhaps best highlighted by the comparatively rapid adoption of ${ }^{99 \mathrm{~m}} \mathrm{Tc}$-based radiopharmaceuticals for myocardial perfusion and the virtual absence of ${ }^{123} \mathrm{I}$ - and ${ }^{111} \mathrm{In}$-based radiopharmaceuticals in clinical practice in Australia. Similarly, the availability of ${ }^{99 \mathrm{~m}} \mathrm{Tc}$ in the southern hemisphere sees a relative favoring of ${ }^{99 \mathrm{~m}} \mathrm{Tc}-$ labeled white blood cells over ${ }^{67} \mathrm{Ga}$ citrate for infection imaging, of ${ }^{99 m} \mathrm{Tc}$-sestamibi over ${ }^{201} \mathrm{Tl}$-chloride in oncology, and of ${ }^{99 \mathrm{~m}} \mathrm{Tc}$-based ventilation agents such as aerosols and Technegas (Cyclopharm) over ${ }^{81 \mathrm{~m}} \mathrm{Kr}$ (which is not clinically available). Nonetheless, at the time this article 
was being written Australia was suffering its own version of a ${ }^{99 \mathrm{~m}} \mathrm{Tc}$ crisis, with the national reactor closed for routine maintenance, causing shockwaves through the nuclear medicine community. Those most significantly affected suffered 2 weeks of lower yields on ${ }^{99} \mathrm{Mo} /{ }^{99} \mathrm{~m} \mathrm{Tc}$ generators (e.g., in one of the worst cases drawn to our attention, the department in question received only $60 \%$ of its anticipated calibrated activity). Furthermore, those relying on centralized pharmacies had dose-capping measures used-for example, a $10 \%$ reduction in dispensed doses across the board. Locally, the shockwaves appeared more severe than the reality because ${ }^{99}$ Mo has enjoyed a large production surplus for many years, with generators routinely topped up and leaving departments counting on larger activities than ordered and paid for. In fact, previous global ${ }^{99 \mathrm{~m}} \mathrm{Tc}$ crises have simply seen Australian departments get the activity ordered and paid for (rather than the higher yields consumers have become accustomed to due to the "topped up" generator activities).

Although the United States enjoys 6.5 PET/CT scanners per million people and Europe has 1.2 PET/CT scan- ners per million people (3), Australia gets by with fewer than $0.5 \mathrm{PET} / \mathrm{CT}$ scanners per million people. Furthermore, states such as Texas, Florida, New York, and Pennsylvania have populations less than that of Australia, yet each has between 10 and 16 times more PET/CT scanners servicing its population. Thus, the relative abundance of ${ }^{99 \mathrm{~m}} \mathrm{Tc}$ might be offset by a relative absence of other novel non- ${ }^{99 m}$ Tc SPECT tracers and scarcity of PET/CT services. Moreover, the bulk of the medical literature globally reflects practice in the northern hemisphere, and the discordance between the hemispheres may not be reflected in the literature. Consequently, the scourge that is the ${ }^{99 \mathrm{~m}} \mathrm{Tc}$ crisis in the northern hemisphere may be perceived as a global issue and change referral patterns and clinical practice unnecessarily in the southern hemisphere. Conversely, the expectation in the southern hemisphere for access to PET/CT services or novel tracers might fall frustratingly short of the actual capabilities.

A comparison of nuclear medicine status and service provision between the 2 hemispheres highlights different capabilities and different barriers, and these are not always reflected in the typically northern hemispheric-driven journal publications. A comparison of nuclear medicine status and service provision between the 2 hemispheres is like comparing apples and oranges, or perhaps baseball and cricket.

\section{Geoffrey M. Currie Hosen Kiat Janelle M. Wheat}

Australian School of Advanced Medicine, Macquarie University, Sydney, Australia

Centre for Research in Complex Systems (CRiCS), Charles Sturt University, Wagga Wagga, Australia

\section{REFERENCES}

1. Guérin B, Tremblay S, Rodrigue S, et al. Cyclotron production of ${ }^{99 \mathrm{~m}} \mathrm{Tc}$ : an approach to the medical isotope crisis. J Nucl Med. 2010;51(4):13N-16N.

2. Pagnanelli RA, Basso DA. Myocardial perfusion imaging with ${ }^{201}$ Tl. J Nucl Med Technol. 2010;38:1-3.

3. Buck AK, Herrmann K, Stargardt T, Dechow T, Krause BJ, Schreyögg J. Economic evaluation of PET and PET/CT in oncology: evidence and methodologic approaches. J Nucl Med Technol. 2010;38:6-17. 